Preventive Care in Nursing and Midwifery Journal

2020; 9(4): 7-14

\title{
Evaluating the Observance Degree of Sterile Principles in The Process of Urinary Catheterization
}

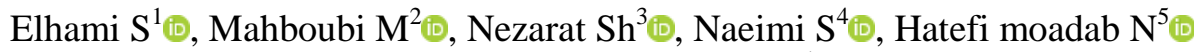 \\ Zahedi $\mathrm{A}^{6}{ }^{\mathbb{D}}$, Zadabdollah $\mathrm{A}^{7^{*}}$ \\ ${ }^{1}$ Instructor, MSc in Medical-Surgical Nursing, Abadan Faculty of Medical Sciences, Abadan, Iran \\ ${ }^{2}$ Associate Professor, PhD in Health Services Management, Abadan Faculty of Medical Sciences, Abadan, Iran \\ ${ }^{3}$ BSc in Nursing, Student Research Committee, Abadan Faculty of Medical Sciences, Abadan, Iran \\ ${ }^{4}$ BSc Student in Nursing, Student Research Committee, Abadan Faculty of Medical Sciences, Abadan, Iran \\ ${ }^{5} \mathrm{Ph} . \mathrm{D}$ Candidate in Nursing, School of Nursing and Midwifery, Tehran University of Medical Sciences, Tehran, Iran \\ ${ }^{6}$ Instructor, MSc in Epidemiology, Abadan Faculty of Medical Sciences, Abadan, Iran \\ ${ }^{* 7}$ BSc in Operating Room Technologist, Student Research Committee, Abadan Faculty of Medical Sciences, Abadan, Iran
}

“Corresponding Author: BSc in Operating Room Technologist, Student Research Committee, Abadan Faculty of Medical Sciences, Abadan, Iran

Tel: 0098- 9168802170

Email: atefeh.zdh@gmail.com

Received: 10 May $2020 \quad$ Accepted: 11 Aug 2020

\begin{abstract}
Background: Urinary tract infections are responsible for $45 \%$ of hospital-acquired infections, of which the urinary catheter is responsible for $80 \%$ of hospital-acquired urinary infections.

Objectives: Due to the importance of the issue and its relationship with the application of correct principles of catheterization in accordance with existing standards in preventing infection, we decided to investigate the observance degree of sterile principles of catheterization in medical wards.

Methods: In this descriptive-analytical study, two researchers in different medical wards of selected hospitals of Abadan University of Medical Sciences evaluated the procedure based on a researcher-made checklist. The data was analyzed by Pearson correlation using SPSS-21 software.

Results: In this study, 150 people were included as the participants. The results of the study indicated better performance in women and people with a history of less than 5 years, and the best performance belonged to the age group of 20-29 years. Besides, based on the position, students and nursing experts achieved the best performance, and the group of Practical Nurse and physicians showed the weakest performance.

Conclusion: The results of the study indicate lack of knowledge or inattention to the observance of standards and their importance among health staff. This especially holds true in people with working experience and older ages, practical nurses and physicians, and those who neglected continuous training and followed the procedures incorrectly. The working principles are mostly performed on a routine basis with the least amount of time and accuracy. Therefore, more attention should be devoted to this issue through workshops and educational forums as well as interaction with centers of evidence-based care that use their life experiences with regard to existing facilities.
\end{abstract}

Keywords: sterile principles, urinary catheter, urinary tract

\section{Introduction}

Urinary tract is the second leading cause of infection in human body and one of the most common bacterial infections among more than 95\% of patients admitted to the hospital and referred to the laboratories [1,2]. Any hospital- acquired urinary infection adds $\$ 676$ to the cost of hospitalization, and when bacteremia occurs, this additional cost reaches to $\$ 2,836$ [3]. In Iran, there are no accurate segregation statistics on nosocomial infection and their specific physical and financial complications. 
Nonetheless, Ghanbari et al. reported $72 \%$ of nosocomial infections as urinary infections [4], and Yaghoubi reported nosocomial infection as the most common form (35.2\%) [5].

Catheterization in urinary tract is one of the most important risk factors in the prevalence of these infections [6]. Factors such as sex, high age, duration of hospitalization and catheterization of perineum and bladder, use of antibiotics in wide rage and underlying diseases, and lack of proper hand-washing in health care providers play important roles in creating nosocomial urinary tract infections [7]. The method to take care of the catheter during and after insertion and use has an important effect on creating or preventing urinary infection caused by catheter. A wrong way of catheter insertion is the most important risk factor of infection, and the highest risk of cathetercaused infection is also related to its insertion time [8].

Since the incidence of these infections leads to an increase in hospitalization time, the use of multiple antibiotics, the incidence of antibiotic resistances for each individual, and an increase in the costs imposed on individuals and hospitals [910], it seems that special attention to this important issue is essential.

The most important people in this field are nurses as caregivers of patients. Unfortunately, previous research indicated that most nurses do not have adequate awareness in this field. Therefore, continuous training is necessary to increase nurses' awareness and consequently reduce nosocomial infections. Developing such training programs would not be effective without careful reviews as well as an analysis of nurses' performance and awareness of controlling nosocomial infections [11]. For this reason, this study investigates the observance degree of sterilization principles during catheterization in different medical wards of selected teaching hospitals of Abadan University of Medical Sciences to take steps in controlling nosocomial infections, especially those caused by the use of urinary catheter.

\section{Methods}

The present study adhered to a descriptiveanalytical design and was conducted in the first six months of 1396. The staff working in the operating room including special (intensive care unit), internal, surgery, and emergency wards of Abadan University of Medical Sciences in Abadan and Khorramshahr cities were considered as the participants of our study. The sample size was calculated using the study of Adib Haji Bagheri et al. and considering $\alpha=0.05, \mathrm{P}=0.63$, and $d=0.08$. Our final sample included 150 people [8].

Sampling was carried out by means of an available method and based on the inclusion criteria which included:

1. agreement of people to participate in the study, 2. Being employed in an operating room or internal, surgical, and emergency wards of Abadan University of Medical Sciences.

3. having a certificate of participation in infectioncontrolling training courses.

Exclusion criteria were the dissatisfaction of the nurses or patients with the presence of observers. In this study, the sample of the study was selective, and the demographic variables included age, sex, workplace department, position, work experience, and type of shift. Moreover, the data collection tool included a researcher-made observational checklist that was prepared based on the reliable sources of the Iranian nursing system organization including clinical nursing methods of Maliheh Sadat Mousavi, principles and techniques of nursing Potter and Perry, and Fundamental nursing [12-16]. This checklist contains 40 questions to check the observance of the correct tips and principles of catheterization of the urinary system (correct perineal prep, wearing sterile gloves, implementation of the procedure by sterile method, and other cases). To determine the validity of the content, the checklist was distributed among ten faculty members of the School of Nursing, and their comments were applied. The agreement between the observers was used to determine the reliability, and to collect data, each samples of the research community was observed by two researchers. To achieve this, the checklist was provided to the second observer, who was at the same level as the first observer was in terms of skill and experience, and was completed simultaneously. The main variable to check the observance of the correct tips and principles of urinary system catheterization in the operating room and special, internal, surgical, and emergency wards as well as the observance degree of the principles was 
determined by the answers in the checklist. Each item on the checklist was assigned a score, and then the mean score of the checklists was calculated. This mean value was then compared with the total score of each checklist, and the result was reported in terms of percentages. The method of simultaneous observation of the two was used to ensure the scientific trust of the work. The two researchers in different shifts referred to the wards, and if this procedure was followed, they observed the method of catheterization based on the items mentioned in the checklist and marked the implementation or nonimplementation of the correct method in the corresponding column. Sampling was continued until the desired size was obtained .The results were analyzed using SPSS software version 21. It should be noted that all ethical considerations were generally noticed including obtaining the code of ethics from Abadan University of Medical Sciences (IR-ABADANUMS.REC.1395. 125) and informed consent from participants (confidentiality, information, possibility of withdrawing participation if desired, and publishing the results of the study).

\section{Results}

In this study, the observance degree of sterile principles was evaluated during catheterization of 150 people working in the operating room and special, internal, surgery, and emergency wards of Abadan University of Medical Sciences in Abadan and Khorramshahr cities. Among the participants, 99(66\%) were females, and 51(33\%) were males. a majority of the participants $(51.3 \%)$ were within the age group of 20-29 years. According to the results, the highest observance of sterile principles during catheterization was related to the age group of 20-29 years. Demographic information is presented in (Table 1).

Table 1: Demographic information of the participants

\begin{tabular}{|c|c|c|c|c|c|}
\hline Variable & Scale & $\begin{array}{l}\text { Number } \\
\text { (Percent) }\end{array}$ & Variable & Scale & $\begin{array}{l}\text { Number } \\
\text { (Percent) }\end{array}$ \\
\hline \multirow{2}{*}{ Sex } & Male & $50(33.3)$ & \multirow{2}{*}{ Shift } & Morning & $132(88)$ \\
\hline & Female & $100(66.7)$ & & Evening & $18(12)$ \\
\hline \multirow{3}{*}{ Age } & 20-29 Years & $77(51.3)$ & \multirow{7}{*}{ Post } & Nurse & $59(39.3)$ \\
\hline & 30-39 Years & $51(34)$ & & Operating Room Technician & $7(4.7)$ \\
\hline & 40 Years And More & $22(14.7)$ & & Bsc In Operating Room & $17(11.3)$ \\
\hline \multirow{4}{*}{$\begin{array}{c}\text { Hospital's } \\
\text { Ward }\end{array}$} & Operation Room & $50(33.3)$ & & Nursing Assistant & $7(4.7)$ \\
\hline & Medical & $36(24)$ & & Doctor & $6(4)$ \\
\hline & Surgical & $22(14.7)$ & & Nursing Student & $40(26.7)$ \\
\hline & Icu, Ccu ,Emergency Department & $42(28)$ & & Operating Room Student & $14(9.3)$ \\
\hline \multirow{3}{*}{$\begin{array}{c}\text { Work } \\
\text { Experience }\end{array}$} & Less Than Years & $101(67.3)$ & \multirow{3}{*}{ Hospital } & Beheshti & $56(37.3)$ \\
\hline & 6-10 Years & $27(18)$ & & Taleghani & $51(34)$ \\
\hline & More Than 10 Years & $22(14.7)$ & & Valiasr & $43(28.7)$ \\
\hline
\end{tabular}

The observance of sterile principles during catheterization showed that some tasks such as washing the genital area with soap liquid and gas were not performed before the procedure. On the other hand, cases such as using perforate or sterile seam, following the correct method of site disinfection, and clamping the catheter, in case of the need to raise the bladder during the transfer of the patient, were among the least cases that were performed (Table 2). 
Table 2: The percentages of the two urinary catheterization observations according to the checklist

\begin{tabular}{|c|c|c|c|}
\hline row & care & yes & no \\
\hline 1 & Examines the patient's record interms of medical instructions to perform the catheterization without difficulty & 98 & 2 \\
\hline 2 & Brings the catheter set and other necessary equipment to the patient's bedside & 98 & 2 \\
\hline 3 & Identifies the patient and explains the steps to the patient & 56.6 & 43.4 \\
\hline 4 & Provides a private environment for the patient & 78.8 & 21.2 \\
\hline 5 & Washes his/her hands & 46.5 & 53.5 \\
\hline 6 & Helps the patient to be in the proper position & 97 & 3 \\
\hline 7 & Provides adequate light & 80.8 & 19.2 \\
\hline 8 & Wears clean gloves & 98 & 2 \\
\hline 9 & $\begin{array}{l}\text { He (she)exposes the genital area, washes the perineal area with soap and gas from top to bottom, } \\
\text { or asks the patient to wash the perineal area himself (herself) if he (she) is able }\end{array}$ & 0 & 100 \\
\hline 10 & Lifts the basin & 0 & 100 \\
\hline 11 & Takes off his gloves & 0 & 100 \\
\hline 12 & Washes his/her hands again & 42.4 & 57.6 \\
\hline 13 & Opens the sterile set correctly. & 66.7 & 33.3 \\
\hline 14 & $\begin{array}{l}\text { Opens the catheter and urinary bag aseptically and places it on the set correctly without colliding with } \\
\text { the inside of the set. }\end{array}$ & 71.7 & 28.3 \\
\hline 15 & Places the softener on a gas inside the set to slide the catheter head correctly & 67.7 & 32.3 \\
\hline 16 & Adds disinfectant solution to cotton or gas pellets properly & 59.6 & 40.4 \\
\hline 17 & Adds the washing serum solution to the cotton or gas pellets properly & 55.6 & 44.4 \\
\hline 18 & Prepares a syringe containing distilled water and place it outside the set & 59.9 & 10.1 \\
\hline 19 & Wears sterile gloves properly. & 52.5 & 47.5 \\
\hline 20 & Places a sterile seam under the patient's buttocks or uses a perfodegreed seam on the patient's body. & 39.2 & 60.8 \\
\hline 21 & Attaches the lower end of the catheter to the urine collection bag & 81.8 & 18.2 \\
\hline 22 & Picks up disinfectant cotton balls with his dominant hand & 93.9 & 6.1 \\
\hline 23 & Properly disinfect the site related to the sex & 29.3 & 70.7 \\
\hline 24 & Uses a new cotton for each cleaning & 43.4 & 56.6 \\
\hline 25 & Repeat the above steps with the washing serum & 25.3 & 74.7 \\
\hline 26 & Removes the dry gas by forceps and dry the rest of the solution. & 49.5 & 50.5 \\
\hline 27 & Inserts the pre-prepared catheter into the urethra approximately 2 inches to allow urine to flow & 88.9 & 11.1 \\
\hline 28 & After observing the flow of urine, he inserts another $2.5 \mathrm{~cm}$ catheter and holds the catheter in a fixed place & 86.9 & 13.1 \\
\hline 29 & Fills the balloon with the dominant hand & 98 & 2 \\
\hline 30 & Gently pulls the catheter out after filling to ensure its location & 94.9 & 5.1 \\
\hline 31 & After making sure, he(she) inserts the catheter in again & 37.4 & 62.6 \\
\hline 32 & With catheter tape, it attaches to the patient's body in the correct way according to the sex & 99 & 1 \\
\hline 33 & Writes the date of the catheter fix on the appropriate label and attaches to it & 91.9 & 8.1 \\
\hline 34 & Fixes the bladder below the patient's body surface & 99 & 1 \\
\hline 35 & Examines urine for volume, color, and abnormalities for recording in the report & 83.8 & 16.2 \\
\hline 36 & Avoids sudden emptying of the bladder and excretes urine alternately by clamping and opening the catheter & 67.7 & 32.3 \\
\hline 37 & If there is a need to raise the bladder during moving the patient, be sure to clamp the catheter & 20.2 & 79.8 \\
\hline 38 & Helps the patient to be in a comfortable position & 97 & 3 \\
\hline 39 & Collects extra items in the correct way & 99 & 1 \\
\hline 40 & Takes off the gloves and washes hands & 80.8 & 19.2 \\
\hline
\end{tabular}

The results of variance analysis regarding the relationship between demographic characteristics and the observance degree of catheterization principles by gender are depicted in Table 3. In terms of working experience, the lowest degree of observance was related to people with more than 10 years of experience, and with regard to position, the lowest degree of observance was related to Practical Nurses. The highest degree of principles observance for men was among operating room experts and for females, was among nursing students. In both sexes, the lowest degree of observance was observed among Practical Nurses, which was statistically significant. There was no significant difference in terms of shift and type of hospital. However, in the case of working experience, only among women, higher work experience was associated with a decrease in the observance degree of the catheterization principles. In addition, regarding 
the workplace, the highest degree of observance was observed in men working in special wards and in women working in the internal ward. And, the lowest degree was found in both males and females in the operating room ward (Table 3).

Table 3: Descriptive statistics of the principles of catheterization used by two genders based on demographic factors

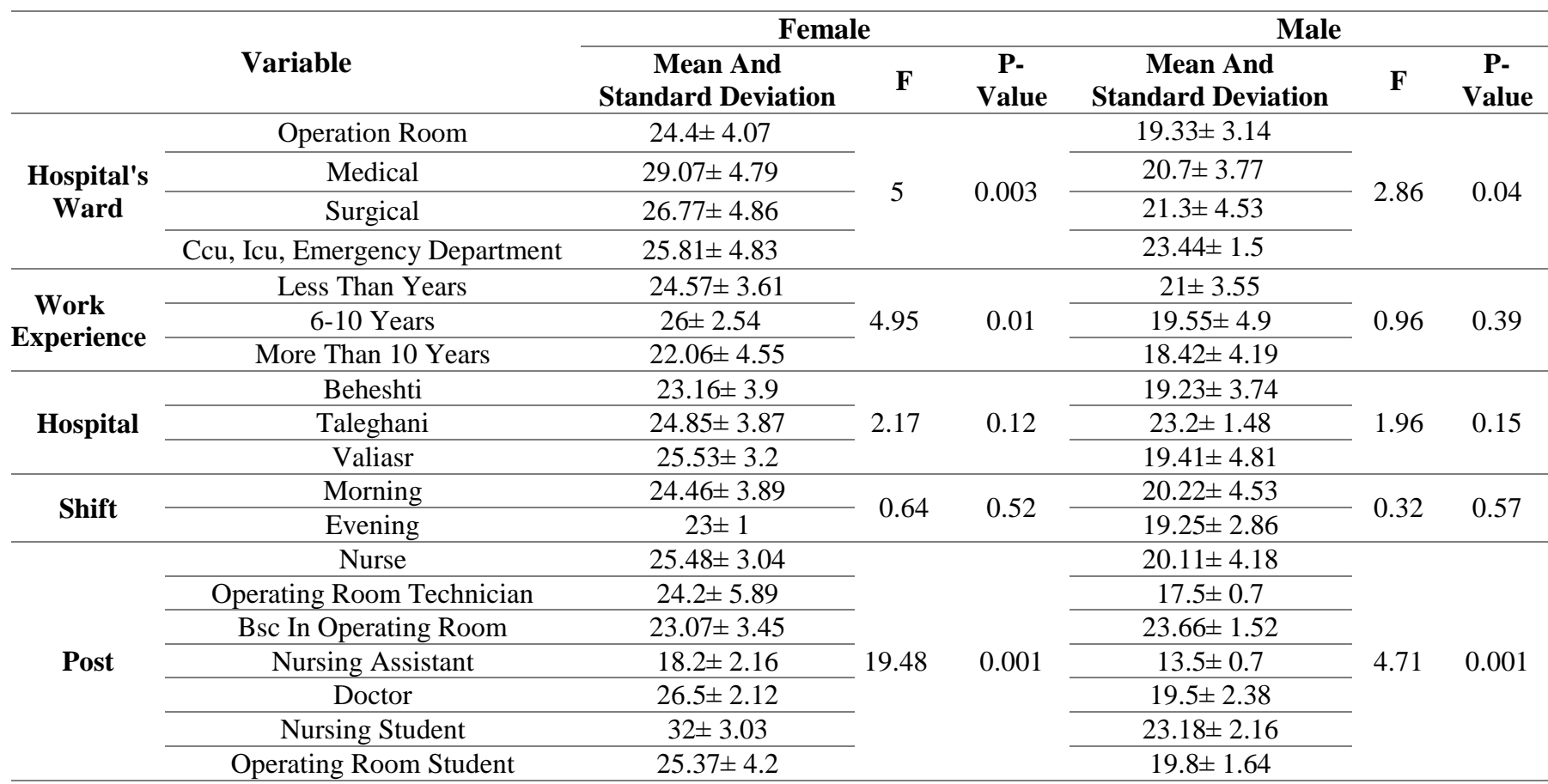

The results of Pearson correlation coefficient on demographic factors showed that in accordance with increasing age and work experience, the observance of catheterization principles decreased in both sexes. In men, no relationship was found between the position and observance of catheterization. In women, this relationship was weak. In relation to the ward, a significant inverse relationship was also observed in men, which was moderate, while this relationship was meaningless in women. Details of the results are provided in (Table 4).

Table 4: Pearson correlation coefficients between demographic factors and the observance degree of catheterization principles in two genders

\begin{tabular}{ccccc}
\hline \multirow{2}{*}{ Variable } & \multicolumn{2}{c}{ Female } & \multicolumn{2}{c}{ Male } \\
\cline { 2 - 5 } & Correlation Coefficient & P-Value & Correlation Coefficient & P-Value \\
\hline Age & 0.48 & 0.0001 & 0.46 & 0.001 \\
\hline Work Experience & 0.44 & 0.0001 & 0.43 & 0.002 \\
\hline Post & 0.25 & 0.01 & -0.13 & 0.35 \\
\hline Workplace Ward & 0.04 & 0.64 & -0.34 & 0.01 \\
\hline
\end{tabular}

\section{Discussion}

The observance of sterile principles during catheterization showed that cases such as washing the genital area with soap liquid and gas were not performed before the procedure. However, cases such as using sterile or perforate seam, following the correct method of site disinfection, and clamping the catheter, in case of the need to raise the bladder during the patient transfer, were among the least cases that were performed (Table 2). Haj Bagheri et al. showed that when the bladder was higher than the level of the bladder, its tube was not clamped in more than half of the cases, and further concluded that the overall quality of patient care with catheters was not good. Explanations to the patients in terms of whether the catheter was fixed in the bladder were not provided in more than $60 \%$ of cases, and the catheter was not fixed outside the bladder in a significant percentage of patients. Besides, necessary trainings were not provided in more than $70-90 \%$ of patients [8]. Those procedures 
that were not performed in the present study have a significant contribution in preventing infections caused by urinary catheter. Observing such cases can indicate a lack of awareness or inattention to the observance of these standards and their importance among health staff. Therefore, planning programs of nursing managers in order to increase the awareness and adaptation of nurses in this field need serious attention. Moreover, due to the educational nature of these hospitals, the role of students in this area cannot be neglected, and all health care providers should be involved in the planning process.

In this study, in addition to the observance degree of urinary catheterization principles, the relationship among some individual factors (gender, work experience, age, and position) was also investigated. A comparison of the observance of sterile principles during catheterization by gender showed that women performance was better than that of men in observing sterile principles during catheterization Table 4. This finding is in line with the study of Teymouri et al. during which infection control standards were performed in Kermanshah in order to investigate the performance of Members of the surgical team. The results showed that women performed better than men in observing infection control standards [17]. However, this difference was not observed in Khodaveisi 'study on the observance degree of infection control standards [18]. The difference in performance in the field of occupational health principles is attributable to the inherent differences between men and women in terms of health care sensitivities.

The comparison of sterile principles observance during catheterization based on work experience showed that those with the work experience of less than 5 years performed better both in women $(69.3 \%)$ and men groups (71.5\%). The degree of observance of correct sterile principles during catheterization decreased in accordance with increasing work experience Tables 3 and 4. This issue was also true for age factor. In the present study, most people were in the age group of 20-29 years, and the observance degree of correct sterile principles during catheterization decreased in line with the increasing age of employees. The results of a study conducted by Majidi et al. on anesthesia personnel to apply the principles of infection control by operating room staff in Rasht
Teaching and Medical hospitals are not in line with the results related to work experience in the present study. However, the results related to age are consistent with our findings. They also concluded that the observance degree of infection control principles decreased with increasing ages [19]. The study of Ansarian et al. pointed out that most people with the age range of 23-25 years old performed better [20]. According to the researchers, the main reason for this can be the ignorance of the increasing age, continuous training, and proper procedures, and the principles of work are carried out more on a routine basis with the least amount of time and accuracy. On the other hand, when when a person is newly employed and has little work experience, points such as recent completion of university education, little time gap between one's education and employment, being young and motivated workforce could lead such individuals to demonstrate more acceptable professional performance and efficiency despite being less experienced. In comparing the observance of sterile principles during catheterization in terms of observers' position, the best performance was found among nursing experts and students in both sexes, and the weakest performance was observed among physicians and Practical Nurses Table 3. However, this relationship was weakly correlated with Pearson correlation. This relation, however, was confirmed in the study of Ansari et al. and Silaji [20,21].

The reason for this matter could be due to the way of learning skills among health care staff. The nurses group is usually academically trained by an instructor in simulation centers (clinical skills center) and then trained for several years during the internship courses under the supervision of their instructors. In contrast, the training of physicians is mostly done in the clinical environment by people with a doctorate degree, those who are spending their residency and training of Practical Nurse experimentally, and traditionally by clinical people who may not be well-trained in related techniques and the sterile of instruments.

The comparison of Pearson correlation coefficients of the observance of sterile principles during catheterization in terms of hospital ward and by gender showed that this relationship was meaningless in women and moderate in men 
Table 4. The present study encountered several limitations. First, we can mention the implementation of this study in only 3 teaching hospitals in Abadan and Khorramshahr cities. Second, the sample size was small, which limits the generalizability of the results. Third, we conducted this study among the staff of different medical wards.

Consequently, it was not possible for us to find the reasons for the incorrect implementation of the guideline to install and take care of urinary catheters. Future research can take these issues to account and find out the possible reasons.

\section{Conclusion}

The results of the study indicated the lack of knowledge or inattention to the observance of standards and their importance among health staff, especially those with working experience and older ages, Practical Nurses and physicians, and men who ignored continuous training and doing proper procedures. Moreover, we found that the principles of work were mostly done on a routine basis with the least amount of time and accuracy. Based on the results of this study and the importance of preventing nosocomial infections, targeted training courses are suggested to be considered during employment, implementation, and evaluation courses which do not just focus on training of school periods. This can be achieved through workshops and educational forums, creating interactions with centers of implementing evidence-based care, and using life experiences with regard to existing facilities.

Holding these courses, monitoring catheterization complications, and providing continuous feedback to health care staff such as doctors and nurses can have a significant impact on the degree of urinary infections and other catheterization complications.

\section{Acknowledgements}

This article is a part of a research plan approved by Abadan faculty of Medical Sciences No. 95st0062. The researchers express their gratitude and appreciation to the Vice Chancellor for Research and Technology of Abadan faculty of Medical Sciences and the esteemed participants in the study.

\section{Conflict of interest}

The author declares no conflicts of interest.

\section{References}

1. Jarsiah P, Alizadeh A, Mehdizadeh E, Ataee R. Khanalipour N. Evaluation of Antibiotic Resistance Model of Escherichia Coli in Urine Culture Samples at Kian Hospital Lab in Tehran, 2011-2012. J Mazandaran Univ Med Sci. 2014; 24(111): 78- 83. [In Persian]

2. Saedi S, Chakerzehi A, Soltani N, Honarmand M, Yazdanpanah M, Ghazvini K. Nosocomial Urinary Tract Infections: Etiology, Risk Factors and Antimicrobial Pattern in Ghaem University Hospital in Mashhad. J Param Sci Rehabili. 2013; 2(1): 22- 25. [In Persian]

3. Azadmanesh Y, Azimian J, Jahani Hashemi H, Shahrokhi A, Naemian Sh. Comparing The Effect of Washing Meatus Urinary Region with Chlorhexidine and Poidon Iodine Solutions on the Appearance of Bacteriuria In Patients with Fixed Urinary Catheter Suffering From Brain Stroke (CVA). Modern Care J. 2013; 10 (2): 241- 48. [In Persian]

4. Ghanbari F, Ghajavand H, Behshod P, Ghanbari N, Khademi F. Prevalence of HospitalAcquired Infections in Hospitalized Patients in Different Wards of Shariati Hospital of Isfahan, 2014. J Health. 2018; 8(5): 511- 17. [In Persian]

5. Yaghubi T, Pourkazemi A, Farashbandi H, Balu H. Epidemiological Study Of Nosocomial Infections and Antibiotic Resistance Patterns in Guilan. Yafte. 2019; 21(1): 52- 62. [In Persian]

6. Mehrabi Tavana A. Nosocomial Infections: A Global Problem. Hakim Health Sys Res. 2016; 19(2): 100- 02. [In Persian]

7. Storme O, Saucedo J.T, Garcia-Mora A, Dehesa-Dávila M, Naber K. Risk Factors and Predisposing Conditions for Urinary Tract Infection. Ther Adv Urol. 2019; 11: 19- 28.

8. Adib Hajbaghery M, Agha Jani M. Quality Of Care For Patients With indewelling Urinary Catheter in University Hospitals of Kashan, 2005. Feyz. 2008; 12(1): 61- 68. [In Persian]

9. Sultan I, Kilic A, Arnaoutakis G, Kilic A. Impact of Foley Catheter Placement By Medical Students on Rates of Postoperative Urinary Tract Infection, J Am Coll Surg. 2018; 227(5): 496501.

10. Zurmehly J. Implementing A Nurse-Driven Protocol to Reduce Catheter-Associated Urinary 
Tract Infections in A Long-Term Acute Care Hospital. J Contin Educ Nurs. 2018; 49(8): 37277.

11. Amini N, Reza Zadeh A, Khoshe Mehri G, Amini M, Salehi Nia H. Knowledge, Attitude And Self-Efficacy of Nursing Staff in Nosocomial Infection in Child Hospitals of Tehran university of Medical Sciences. Alborz Uni Med Sci J. 2015; 4(1): 27- 34. [In Persian]

12. Mousavi M, Alikhani M. Clinical Nursing Techniques And Examination of Physical Health Status. $7^{\text {th }}$ ed. Tehran: Shahre Pub; 2018. 205- 11. [In Persian]

13. Crisp J, Douglas C, Rebeiro G, Waters D. Potter \& Perry's Fundamentals of Nursing. $5^{\text {th }}$ ed. Australia, Chatswood: Elsevier; 2017.

14. Berban A, Snyder Sh, Frandsen G. Kozier \& Erb's Fundamentals of Nursing: Concepts, Process, and Practice. $10^{\text {th }}$ ed. 2015.

15. Kuhn Timby B. Fundamental Nursing Skills and Concepts. $11^{\text {th }}$ ed. Philadelphia: Lippincott Williams\& Wilkins; 2016: 723- 33.

16. Lyn P. Taylor's Fundamentals of Nursing. Sharifi Nodehi $M$ [Translator]. $5^{\text {th }}$ ed. Tehran: Boshra; 2019

17. Teimoori B, Ghafari P, Veisi Raigan A, et al. Surgery Team Members' Performance With
Regard To Observing Infection Control Principles in Educational Centers Operation Rooms of KUMS. J Kermanshah Univ Med Sci. 2004; 8(1): 51- 61. [In Persian]

18. Khodaveisi M, Mohamadkhani M, Amini R, Karami M. Factors Predicting The Standard Precautions for Infection Control Among PreHospital Emergency Staff of Hamadan Based on the Health Belief Model. J Educ Community Health. 2017; 4(3): 12- 18. [In Persian]

19. Magidi SA, Mehrabian F, Tabari R. Accomplish Principles of Infection Control by Operation Room's Staff in the Rasht Hospitals. J Guilan Univ Med Sci. 2008; 16(64): 89- 96. [In Persian]

20. Ansarian N, Hashemi F. Evaluation of The Principles of Sterile Catheterization in The Operating Room Teaching Hospitals of Qazvin Province in 2009. Edrak J Qazvin Univ Res Committee. 2012; 7(26): 49- 52. [In Persian] http://vcr.qums.ac.ir/Portal/file/?126956/268.pdf. 21. Szilágyi L, Haidegger T, Lehotsky Á, Nagy M, Csonka E, Sun X. A Large-Scale Assessment of Hand Hygiene Quality and the Effectiveness of The "WHO 6-Steps". BMC Infect Dis. 2013; 13: 249. 